\title{
Signed Excedance Enumeration in the Hyperoctahedral group
}

\author{
Sivaramakrishnan Sivasubramanian \\ Department of Mathematics \\ Indian Institute of Technology, Bombay, India
}

krishnan@math.iitb.ac.in

Submitted: Jul 6, 2013; Accepted: Mar 30, 2014; Published: Apr 16, 2014

Mathematics Subject Classifications: 05A05, 05A30, 05A19

\begin{abstract}
Several signed excedance-like statistics have nice formulae or generating functions when summed over the symmetric group and over its subset of derangements. We give counterparts of some of these results when we sum over the hyperoctahedral group and its subset of derangements. Our results motivate us to define and derive attractive bivariate formulae which generalise some of these results for the symmetric group.
\end{abstract}

Keywords: Signed excedance; permutation group; hyperoctahedral group

\section{Introduction}

For a positive integer $n$, let $[n]=\{1,2, \ldots, n\}$ and let $\mathfrak{S}_{n}$ be the set of permutations on $[n]$. Let $\mathfrak{B}_{n}$ be the set of permutations $\sigma$ of $\{-n,-(n-1), \ldots,-1,1,2, \ldots n\}$ satisfying $\sigma(-i)=-\sigma(i)$. Clearly any such $\sigma$ is well defined when given $\sigma(i)$ for $i \in[n]$. $\mathfrak{B}_{n}$ is referred to as the hyperoctahedral group or the group of signed permutations on $[n]$, though we do not need its group structure in this work. Clearly, $\left|\mathfrak{S}_{n}\right|=n$ ! and $\left|\mathfrak{B}_{n}\right|=$ $2^{n} n$ !. For $\sigma \in \mathfrak{B}_{n}$, from now on we denote $\sigma(i)$ instead as $\sigma_{i}$. For $1 \leqslant k \leqslant n$, we also denote $-k$ alternatively as $\bar{k}$.

Let $\pi=\left(\pi_{1}, \pi_{2}, \ldots, \pi_{n}\right) \in \mathfrak{S}_{n}$. Define its excedance set, ExcSet $(\pi)$ as $\left\{i \in[n]: \pi_{i}>i\right\}$ and its number of excedances as $\operatorname{exc}(\pi)=|\operatorname{ExcSet}(\pi)|$. For $\pi \in \mathfrak{S}_{n}$, define its number of inversions as $\operatorname{inv}_{\mathrm{A}}(\pi)=\left|\left\{1 \leqslant i<j \leqslant n: \pi_{i}>\pi_{j}\right\}\right|$ and its $n$-th position index pos_n $(\pi)$ as the index $i \in[n]$ such that $\pi_{i}=n$.

For a positive integer $n \geqslant 1$, define the signed excedance enumerator as $\operatorname{SgnExc}_{n}(q)=$ $\sum_{\pi \in \mathfrak{S}_{n}}(-1)^{\operatorname{inv}_{A}(\pi)} q^{\operatorname{exc}(\pi)}$. If $\mathfrak{D}_{n}$ is the set of derangements on $[n]$, and if the number of signed derangements is defined as $\operatorname{SgnDer}_{n}=\sum_{\pi \in \mathfrak{D}_{n}}(-1)^{\operatorname{inv}_{A}(\pi)}$, then it is known that 
$\operatorname{SgnDer}_{n}=(-1)^{n-1}(n-1)$ (see Sivasubramanian [5, Remark 5]). We recall that for a non-negative integer, $i$, its $q$-analogue is defined as $[i]_{q}=1+q+q^{2}+\cdots+q^{i-1}$, where $q$ is an indeterminate and $[0]_{q}=0$. For $n \geqslant 1$, define $\operatorname{DSgnExc}_{n}(q)=\sum_{\pi \in \mathfrak{D}_{n}}(-1)^{\operatorname{inv}_{\mathrm{A}}(\pi)} q^{\operatorname{exc}(\pi)}$ as the signed excedance enumerator over derangements.

In this work, we enumerate signed excedances in $\mathfrak{B}_{n}$. Our motivation comes from the following two beautiful results in $\mathfrak{S}_{n}$.

Theorem 1 (Mantaci [3]) Let $n \geqslant 1$ be a positive integer. Then

$$
\operatorname{SgnExc}_{n}(q)=(1-q)^{n-1} \text {. }
$$

Theorem 2 (Mantaci and Rakotondrajao [4]) Let $n \geqslant 1$ be a positive integer. Then

$$
\operatorname{DSgnExc}_{n}(q)=(-1)^{n-1} q \cdot[n-1]_{q} \text {. }
$$

The original proofs of both results used sign reversing involutions and both papers prove more detailed results as well. An alternate proof of both these results was given by Sivasubramanian in [5], where both $\operatorname{SgnExc}_{n}(q)$ and $\operatorname{DSgnExc}_{n}(q)$ were found out to be determinants of suitably defined $n \times n$ matrices. The connection between determinants and signed excedance enumeration is a simple consequence of Leibniz's formula for the determinant. Further, in [5], several other known and new excedance type statistics in $\mathfrak{S}_{n}$ were also enumerated with signs, by evaluating determinants of similar $n \times n$ matrices. In this work, we give counterparts of some of the results of [5] in the case when enumeration is done over $\mathfrak{B}_{n}$, the set of signed permutations. Since we do not have a determinant-type expansion involving a signed sum over elements of the hyperoctahedral group, our proofs use sign-reversing involutions on $\mathfrak{B}_{n}$.

It is easy to see that $\mathfrak{B}_{n}$ can be considered as a subgroup of $\mathfrak{S}_{2 n}$. Thus, it would be very interesting if the results in this paper on $\mathfrak{B}_{n}$ can be obtained by evaluating the determinant of a $2 n \times 2 n$ matrix, or even the determinant of a matrix with order polynomial in $n$, as done in [5].

Our proof of these results motivate us to define bivariate signed excedance enumerators in $\mathfrak{B}_{n}$ and hence in $\mathfrak{S}_{n}$ as well. Over $\mathfrak{S}_{n}$, these bivariate versions give a sharpening of several known results including Theorem 1 and Theorem 2 (see Theorems 10 and 24 respectively).

Several definitions of excedance exist in $\mathfrak{B}_{n}$ and we follow Brenti's definition from [1], which we recall. For $\sigma=\left(\sigma_{1}, \sigma_{2}, \ldots, \sigma_{n}\right) \in \mathfrak{B}_{n}$, define $\operatorname{ExcSet}(\sigma)=\left\{i \in[n]: \sigma_{\left|\sigma_{i}\right|}>\right.$ $\left.\sigma_{i}\right\} \cup\left\{i \in[n]: \sigma_{i}=-i\right\}$ and let $\operatorname{exc}(\sigma)=|\operatorname{ExcSet}(\sigma)|$. We next give the definition of inversions in $\mathfrak{B}_{n}$. For $\sigma \in \mathfrak{B}_{n}$, let $\operatorname{NegSet}(\sigma)=\left\{i \in[n]: \sigma_{i}<0\right\}$ be the set of indices where $\sigma$ takes negative values and let $\operatorname{nsum}(\sigma)=-\left(\sum_{i \in \operatorname{NegSet}(\sigma)} \sigma_{i}\right)$ be the absolute value of the sum of the negative components of $\sigma$. Define the number of type-A inversions of $\sigma$, as before as $\operatorname{inv}_{\mathrm{A}}(\sigma)=\left|\left\{1 \leqslant i<j \leqslant n: \sigma_{i}>\sigma_{j}\right\}\right|$. Here, comparison is done with respect to the standard order on $\mathbb{Z}$. Define the number of inversions of $\sigma \in \mathfrak{B}_{n}$ as $\operatorname{inv}_{\mathrm{B}}(\sigma)=\operatorname{nsum}(\sigma)+\operatorname{inv}_{\mathrm{A}}(\sigma)$. This combinatorial definition of inversions in $\mathfrak{B}_{n}$ is also due to Brenti (see [1, Proposition 3.1]). If $\sigma \in \mathfrak{B}_{n}$, define pos_n $(\sigma)$ as the index $i \in[n]$ 
such that $\left|\sigma_{i}\right|=n$ and pos_1 $(\sigma)$ as the index $i \in[n]$ such that $\left|\sigma_{i}\right|=1$. In some of our proofs, we need the cycles of $\sigma \in \mathfrak{B}_{n}$. This is an alternate view of $\sigma$ and one can go from the one-line notation of $\sigma$ to its cycle notation and vice versa. We define the cycle notation and give an example of it below. Given $\sigma \in \mathfrak{B}_{n}$, we form a disjoint set of cycles $\mathcal{C}_{\sigma}=\left\{C_{k}\right\}_{k \in P_{\sigma}}$ where $P_{\sigma}$ is some index set such that each $\sigma_{i}$ for $i \in[n]$ is contained in exactly one cycle $C \in \mathcal{C}_{\sigma}$. Each $C$ is formed from the one-line notation of $\sigma$ as follows. We write the elements of $C$ sequentially and if $C$ has elements $\left(x_{1}, x_{2}, \ldots, x_{r}\right)$, then in the one-line notation, we must have had $\sigma_{\left|x_{i}\right|}=x_{i+1}$ where $x_{r+1}=x_{1}$. For example, given the one-line notation of $\sigma=(4, \overline{1}, 3,6,2, \overline{5}) \in \mathfrak{B}_{6}$, its cycle notation is $\mathcal{C}_{\sigma}=\{(\overline{1}, 4,6, \overline{5}, 2) ;(3)\}$. To get the one-line notation of $\sigma$ given $\mathcal{C}_{\sigma}$, we need to get $\sigma_{i}$ for each $i \in[n]$. For each $i \in[n]$, either $i$ or $\bar{i}$ will be present in exactly one of the cycles $C_{r} \in \mathcal{C}_{\sigma}$. Let $i$ occur in $C_{r}$ with sign $\epsilon$ where $\epsilon= \pm 1 . \sigma_{i}$ is the element succeeding $\epsilon i$ in $C_{r}$ with the sign of the succeeding element taken into account. Though we write the elements of $C_{r}$ in a sequence, as $C_{r}$ is a cycle the first element of $C_{r}$ is the succeeding element of the last element of $C_{r}$.

Let $\sigma \in \mathfrak{B}_{n}$ and $\pi \in \mathfrak{S}_{n}$. We carefully distinguish between $\operatorname{inv}_{\mathrm{B}}(\sigma)$ and $\operatorname{inv}_{\mathrm{A}}(\pi)$, but make no such distinction between $\operatorname{exc}(\sigma)$ and $\operatorname{exc}(\pi)$, or between pos_n $(\sigma)$ and pos_n $(\pi)$, though the definitions are different. This will not cause any problem.

Using Brenti's definition of excedance, Chen, Tang and Zhao [2] show a binomial type equation that the type-B Eulerian polynomial and the type-B derangement polynomial enumerated by excedance satisfy, in the univariate unsigned case. In Subsection 5.1, we show that a very similar binomial type equation is satisfied by our signed bivariate analogue when the exponent of $t$ in the term corresponding to $\sigma$ is either pos_n $(\sigma)$ or pos_1 $(\sigma)$, see Corollaries 25 and 26 .

\section{The involution and a few lemmas}

We begin by proving a few lemmas about a sign-reversing involution which we use frequently. We first define the involution. Let $\sigma=\left(\sigma_{1}, \sigma_{2}, \ldots, \sigma_{n}\right) \in \mathfrak{B}_{n}$ and let $r$ be an index where $1 \leqslant r \leqslant n$. We define an involution $\tau_{r}: \mathfrak{B}_{n} \rightarrow \mathfrak{B}_{n}$. For ease of notation, we write $\tau_{r}(\sigma)=\psi$ and $\psi=\left(\psi_{1}, \psi_{2}, \ldots, \psi_{n}\right)$. The involution is as follows: if $\sigma_{r}=k>0$, define $\psi_{r}=\bar{k}$ and if $\sigma_{r}=\bar{k}<0$, let $\psi_{r}=k$. For indices $i \neq r$, define $\psi_{i}=\sigma_{i}$. That is, $\tau_{r}$ negates the content at index $r$ and leaves the content at other indices unchanged. It is evident that $\tau_{r}$ is an involution for all $1 \leqslant r \leqslant n$. We prove the following property of $\tau_{r}$.

Lemma 3 For all $1 \leqslant r \leqslant n$ and $\sigma \in \mathfrak{B}_{n}$, $\operatorname{inv}_{\mathrm{B}}(\sigma) \not \equiv \operatorname{inv}_{\mathrm{B}}\left(\tau_{r}(\sigma)\right)(\bmod 2)$.

Proof: $\quad$ Fix $r$ and denote $\tau_{r}(\sigma)$ as $\psi=\left(\psi_{1}, \psi_{2}, \ldots, \psi_{n}\right)$. We assume $\sigma_{r}=k>0$ without loss of generality. Consider the set of indices $S=\left\{i:\left|\sigma_{i}\right|<k\right\}$. Clearly, $|S|=k-1$. Denote the indices with elements of $S$ occuring before position $r$ as $S_{1}$. i.e. $S_{1}=\left\{1 \leqslant i<r:-k<\sigma_{i}<k\right\}$. Similarly define $S_{2}=\left\{r \leqslant i \leqslant n:-k<\sigma_{i}<k\right\}$. Thus, $\left|S_{1}\right|+\left|S_{2}\right|=k-1$. Recall $\operatorname{inv}_{\mathrm{B}}(\sigma)=\operatorname{nsum}(\sigma)+\operatorname{inv}_{\mathrm{A}}(\sigma)$. To show that $\operatorname{inv}_{\mathrm{B}}(\sigma) \operatorname{and}_{\operatorname{inv}}(\psi)$ have opposite parity, we count their differences. As $\sigma_{r}>0$, there is no contribution to $\operatorname{nsum}(\sigma)$ and there are $\left|S_{2}\right|$ type-A inversions due to $\sigma_{r}(=k)$. As $\psi_{r}=\bar{k}<0$, we have $k$ 
added to nsum $(\psi)$ and there exist $\left|S_{1}\right|$ type-A inversions in $\psi$ that do not occur in $\sigma$. As these are the only differences, $\operatorname{inv}_{\mathrm{B}}(\sigma)-\operatorname{inv}_{\mathrm{B}}(\psi)=\left|S_{2}\right|-\left(k+\left|S_{1}\right|\right)=-1-2\left|S_{1}\right|$ which is odd, completing the proof.

Just as $\tau_{r}$ negates index $r$, we need involutions $\tau_{S}$ for $S \subseteq[n]$, which simultaneously negate all the indices in $S$ and leave the contents indexed by $[n]-S$ unchanged. By iterating the above lemma, we get the following simple corollary whose proof we omit.

Corollary 4 Let $\sigma \in \mathfrak{B}_{n}$ and let $S \subseteq[n]$. Then, $\operatorname{inv}_{\mathrm{B}}\left(\tau_{S}(\sigma)\right) \equiv(-1)^{|S|} \cdot \operatorname{inv}_{\mathrm{B}}(\sigma)(\bmod 2)$.

We move to our next lemma about excedances. We need the following definitions before we state the lemma. For $\sigma \in \mathfrak{B}_{n}$, $\operatorname{define}_{\operatorname{ExcSet}}(\sigma)=\left\{i \in[n]: \sigma_{|\sigma(i)|}>\right.$ $\left.\sigma_{i}\right\}$, NegFixPtSet $(\sigma)=\left\{i \in[n]: \sigma_{i}=-i\right\}$ and $\operatorname{FixPtSet}(\sigma)=\left\{i \in[n]: \sigma_{i}=i\right\}$. Let $\operatorname{fix}(\sigma)=|\operatorname{FixPtSet}(\sigma)|$. Clearly, $\operatorname{ExcSet}(\sigma)=\operatorname{ExcSet}_{\mathrm{A}}(\sigma) \cup \operatorname{NegFixPtSet}(\sigma)$ is the set of indices where excedances occur in $\sigma$ and $\operatorname{exc}(\sigma)=|\operatorname{ExcSet}(\sigma)|$. For $\sigma \in \mathfrak{B}_{n}$, define $\mathbf{W k N o n E x c S e t}(\sigma)=[n]-\operatorname{ExcSet}(\sigma), \operatorname{wknexc}(\sigma)=|\operatorname{WkNonExcSet}(\sigma)|$. Define $\operatorname{NonExcSet}(\sigma)=\mathrm{WkNonExcSet}(\sigma)-\operatorname{FixPtSet}(\sigma)$ and $\operatorname{nexc}(\sigma)=|\operatorname{NonExcSet}(\sigma)|$.

Lemma 5 Let $\sigma \in \mathfrak{B}_{n}$ and let $C$ be a cycle of $\sigma$ with at least two elements in it. Let $m \in C$ be the minimum element of $C$ in absolute value (i.e. among the elements $x \in C$, $m$ has least $|x|$ value). Let $\left|\sigma_{r}\right|=|m|$ and let $\psi=\tau_{r}(\sigma)$ (i.e. $\psi$ is the same as $\sigma$ except for the sign of $m)$. Then, $\operatorname{ExcSet}(\psi)=\operatorname{ExcSet}(\sigma)$ and thus $\operatorname{exc}(\psi)=\operatorname{exc}(\sigma)$. Further, $\operatorname{wknexc}(\sigma)=\operatorname{wknexc}(\psi)$ and $\operatorname{nexc}(\sigma)=\operatorname{nexc}(\psi)$.

Proof: Assume that $m$ occurs in $\sigma$ with positive sign (the other case is proved identically). Since $m$ is not in a cycle of length one, it has both a successor $\operatorname{succ}(m)$ defined as $\operatorname{succ}(m)=\sigma_{m}$ and a predecessor $\operatorname{pred}(m)$ defined as $\operatorname{pred}(m)=\sigma^{-1}(m)$ and for brevity denote $k=\operatorname{pred}(m)$. Define $\psi=\tau_{k}(\sigma)$ (i.e. $\psi$ is identical to $\sigma$ with the sole difference being that it has opposite sign for $m$ ). Clearly in both $\sigma$ and $\psi$ one of the possibilities for a type-B excedance, namely an index $i$ such that $\sigma_{i}=-i$ will never occur at $r$ (as $\sigma_{r}=m$ and $\left.\psi_{r}=\bar{m}\right)$. Since $m$ is the absolute value-wise smallest element of $C$ in $\sigma$, if there is an excedance due to $\operatorname{pred}(m)$ and $m$ in $\sigma$, then there will be an excedance between $\operatorname{pred}(\bar{m})$ and $\bar{m}$ in $\psi$ and vice versa. Likewise, there will be an excedance in $\sigma$ between $m$ and $\operatorname{succ}(m)$ iff there is an excedance between $\bar{m}$ and $\operatorname{succ}(\bar{m})$.

The argument above shows that $\operatorname{ExcSet}_{\mathrm{A}}(\sigma)=\operatorname{ExcSet}_{\mathrm{A}}(\psi)$. Further, since the sign of $m$ is the only difference between $\sigma$ and $\psi, \operatorname{NegFixPtSet}(\sigma)=\operatorname{NegFixPtSet}(\psi)$. Thus we have $\operatorname{ExcSet}(\sigma)=\operatorname{ExcSet}(\psi)$ and so $\operatorname{exc}(\sigma)=\operatorname{exc}(\psi)$. This implies that WkNonExcSet $(\sigma)=$ WkNonExcSet $(\psi)$. and thus $w \operatorname{knexc}(\sigma)=w \operatorname{knexc}(\psi)$. It is also clear that $\operatorname{FixPtSet}(\sigma)=$ $\operatorname{FixPtSet}(\psi)$ and hence $\operatorname{nexc}(\sigma)=\operatorname{nexc}(\psi)$, completing the proof.

Let $\sigma \in \mathfrak{B}_{n}$ for $n \geqslant 3$ have $r$ as a fixed point (i.e. $\sigma_{r}=r$ ) for some $1 \leqslant r \leqslant n$. Define $\rho \in \mathfrak{B}_{n-1}$ as follows: delete the occurence of $r$ in $\sigma$ and then replace $i$ by $i-1$ for all $r+1 \leqslant i \leqslant n$ with signs preserved (i.e. $\overline{(k+1)}$ will get changed to $\bar{k}$ if $k \geqslant r$ ). We will use this operation later on and for brevity, say $\rho$ is obtained from $\sigma$ by "shrinking $\sigma$ on $[r, n]$ ". It is clear that the cycles of $\rho$ are identical to the cycles of $\sigma$ with just the following changes: the fixed point $r$ of $\sigma$ is removed and for $i \geqslant r$, all elements $i+1$ get 
changed to $i$ at their respective places in the cycles of $\sigma$ with the $i$ 's in $\rho$ having the sign of $(i+1)$ 's in $\sigma$.

Lemma 6 Let $\sigma \in \mathfrak{B}_{n}$ have $r$ as a fixed point and let $\rho \in \mathfrak{B}_{n-1}$ be obtained by deleting $\sigma_{r}$ and then shrinking $\sigma$ on $[r, n]$. Then, $\operatorname{inv}_{\mathrm{B}}(\sigma) \equiv \operatorname{inv}_{\mathrm{B}}(\rho)(\bmod 2), \operatorname{exc}(\sigma)=\operatorname{exc}(\rho)$, $\operatorname{wknexc}(\sigma) \not \equiv \operatorname{wknexc}(\rho)(\bmod 2)$ and $\operatorname{nexc}(\sigma)=\operatorname{nexc}(\rho)$.

Proof: We first prove that $\operatorname{inv}_{\mathrm{B}}(\sigma) \equiv \operatorname{inv}_{\mathrm{B}}(\rho)(\bmod 2)$. All elements to the left of $r$ (i.e. all $\sigma_{k}$ for $k<r$ ) that are larger than $r$ will contribute to $\operatorname{inv}_{\mathbf{A}}(\sigma)$ but not to $\operatorname{inv}_{\mathrm{A}}(\rho)$. Similarly, all elements smaller than $r$ to its right (i.e. all $\sigma_{k}$ for $k>r$ ) will contribute to $\operatorname{inv}_{\mathbf{A}}(\sigma)$ but not to $\operatorname{inv}_{\mathbf{A}}(\rho)$. Likewise, if there are $p$ negative elements $\sigma_{i_{1}}, \sigma_{i_{2}}, \ldots, \sigma_{i_{p}}$ with absolute value larger than $r$, they will contribute $s=\sum_{j=1}^{p}\left|\sigma_{i_{j}}\right|$ to nsum $(\sigma)$. In $\rho$, they will contribute $s-p$ to nsum $(\rho)$.

Let $X=\left\{j: j<r\right.$ and $\left.\sigma_{j}>r\left(=\sigma_{r}\right)\right\}$ be the set of indices smaller than $r$ whose image under $\sigma$ is larger than $r$ and let $\alpha=|X|$. Similarly, let $Y=\left\{j: j>r\right.$ and $\left.\sigma_{j}<r\right\}$ be the set of indices larger than $r$ whose image under $\sigma$ is smaller than $r$ and let $\beta=|Y|$. Let $Z=\left\{j: 1 \leqslant j \leqslant n: \sigma_{j}<0\right.$ and $\left.\left|\sigma_{j}\right|>r\right\}$ and let $\gamma=|Z|$.

From the argument in the first paragraph, the difference between $\operatorname{inv}_{\mathrm{B}}(\sigma)$ and $\operatorname{inv}_{\mathrm{B}}(\rho)$ is $\alpha+\beta+\gamma$. We show that $\alpha+\beta+\gamma \equiv 0(\bmod 2)$. To see this, let $d$ be the number of negative elements in $\sigma$. We first consider the case when $d=0$. Recall $\sigma_{r}=r$. As there are $\alpha$ elements to the left of index $r$ that are larger, there are $(r-1-\alpha)$ elements to $r$ 's left that are smaller than $r$. Thus there are $\alpha$ elements to the right of $r$ that are smaller than $r$. Hence, $\beta=\alpha$. Since there are no negative elements, $\gamma=0$. Thus $\alpha+\beta+\gamma=2 \alpha \equiv 0$ $(\bmod 2)$. We record this as follows:

$$
\text { when } \operatorname{NegSet}(\sigma)=\operatorname{NegSet}(\rho)=\emptyset, \operatorname{inv}_{\mathbf{B}}(\sigma) \equiv \operatorname{inv}_{\mathbf{B}}(\rho)(\bmod 2)
$$

Hence, when $\sigma$ has no negative elements and if $\sigma_{r}=r$, the $\rho$ obtained by shrinking $\sigma$ on $[r, n]$ has the same sign as $\sigma$. Moving onto the general case, if $\sigma$ has $d$ elements that are negative, then let $|\sigma| \in \mathfrak{B}_{n}$ be the signed permutation obtained by replacing $\sigma_{i}$ by $\left|\sigma_{i}\right|$ for all $i$, and likewise obtain $|\rho|$ from $\rho$. By Corollary 4 , $\operatorname{inv}_{\mathrm{B}}(\sigma) \equiv(-1)^{d} \operatorname{inv}_{\mathrm{B}}(|\sigma|)(\bmod$ $2)$ and $\operatorname{inv}_{\mathrm{B}}(\rho) \equiv(-1)^{d} \operatorname{inv}_{\mathrm{B}}(|\rho|)(\bmod 2)$. Since both $|\sigma|$ and $|\rho|$ have no zero elements, by $(1)$, we get $\operatorname{inv}_{\mathrm{B}}(|\sigma|) \equiv \operatorname{inv}_{\mathrm{B}}(|\rho|)(\bmod 2)$. Thus, $\operatorname{inv}_{\mathrm{B}}(\sigma) \equiv \operatorname{inv}_{\mathrm{B}}(\rho)(\bmod 2)$.

From the cycles of $\sigma$ and $\rho$, it is easy to see that $\operatorname{exc}(\sigma)=\operatorname{exc}(\rho)$ and since wknexc $(\sigma)=$ $n-\operatorname{exc}(\sigma)$ and $\operatorname{wknexc}(\rho)=n-1-\operatorname{exc}(\rho)$, it is clear that they have opposite parities. Since $\operatorname{fix}(\sigma)=\operatorname{fix}(\rho)+1$, we get $\operatorname{nexc}(\sigma)=n-\operatorname{exc}(\sigma)-\operatorname{fix}(\sigma)-1=\operatorname{nexc}(\rho)$, completing the proof.

Lemma 7 Let $\sigma \in \mathfrak{B}_{n}$ have $\sigma_{r}=\bar{r}$ and let $\rho \in \mathfrak{B}_{n-1}$ be obtained by deleting $\bar{r}$ and then shrinking $\sigma$ on $[r, n]$. Then, $\operatorname{inv}_{\mathrm{B}}(\sigma) \not \equiv \operatorname{inv}_{\mathrm{B}}(\rho)$ (mod 2). Further, $\operatorname{exc}(\sigma)=\operatorname{exc}(\rho)+1$, $\operatorname{wknexc}(\sigma)=\operatorname{wknexc}(\rho)$ and $\operatorname{nexc}(\sigma) \equiv \operatorname{nexc}(\rho)(\bmod 2)$.

Proof: Obtain $\psi$ from $\sigma$ by changing the sign of $r$ (thus $\psi_{r}=r$ ). By Lemma 3, $(-1)^{\operatorname{inv}_{\mathrm{B}}(\psi)} \not \equiv(-1)^{\operatorname{inv}_{\mathrm{B}}(\sigma)}(\bmod 2)$ and by Lemma 6 we get $(-1)^{\operatorname{inv}_{\mathrm{B}}(\psi)} \equiv(-1)^{\operatorname{inv}_{\mathrm{B}}(\rho)}(\bmod$ $2)$. It is easy to see that $\operatorname{exc}(\sigma)=\operatorname{exc}(\rho)+1$ and $\operatorname{wknexc}(\sigma)=\operatorname{wknexc}(\rho)$. Moreover, as $\operatorname{fix}(\sigma)=\operatorname{fix}(\rho)$, we get $\operatorname{nexc}(\sigma) \equiv \operatorname{nexc}(\rho)(\bmod 2)$, completing the proof. 


\section{Signed excedance enumeration in $\mathfrak{B}_{n}$}

For $n \geqslant 1$ and $1 \leqslant i \leqslant n$, let $\operatorname{BSgnExc}_{n}^{i}(q, t)=\sum_{\sigma \in \mathfrak{B}_{n}}(-1)^{\operatorname{inv}_{\mathrm{B}}(\sigma)} q^{\operatorname{exc}(\sigma)} t^{\text {pos_i }(\sigma)}$ be a bivariate signed enumerator counting excedance and the index where $i$ up to sign occurs. Define $\operatorname{BSgnExc}_{0}^{0}(q, t)=1$. We begin with the following bivariate analogue of Theorem 1 to the hyperoctahedral group.

Theorem 8 For $n \geqslant 1$ and $1 \leqslant i \leqslant n, \operatorname{BSgnExc}_{n}^{i}(q, t)=t^{i}(1-q)^{n}$.

Proof: The result can be readily checked when $n=1,2$ and so we assume $n \geqslant 3$. Let $X_{i}=\left\{\sigma \in \mathfrak{B}_{n}\right.$ : pos_i $\left.(\sigma)=i\right\}$. Consider the map $\tau_{k}:\left(\mathfrak{B}_{n}-X_{i}\right) \mapsto\left(\mathfrak{B}_{n}-X_{i}\right)$ defined as follows: let $\sigma \in \mathfrak{B}_{n}-X_{i}$. Since $i$ is not in a cycle by itself, it is in a cycle $C$ of length at least two. Let $m$ be the minimum element of $C$ in absolute value and let $|\sigma(k)|=|m|$. Define $\psi=\tau_{k}(\sigma)$. Thus in $\psi, m$ occurs with opposite sign as compared to $\sigma$. By Lemma $3, \operatorname{inv}_{\mathrm{B}}(\psi) \not \equiv \operatorname{inv}_{\mathrm{B}}(\sigma)(\bmod 2)$ and by Lemma $5, \operatorname{exc}(\psi)=\operatorname{exc}(\sigma)$. Further,

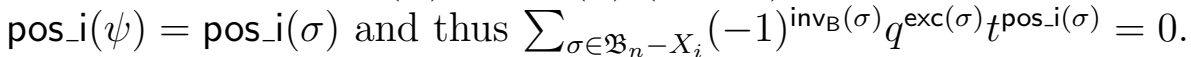

Thus, BSgnExc ${ }_{n}^{i}(q, t)=\sum_{\sigma \in X_{i}}(-1)^{\operatorname{inv}_{\mathrm{B}}(\sigma)} q^{\operatorname{exc}(\sigma)} t^{\text {pos_i }(\sigma)}$. We can thus pull out $t^{i}$ from each term in the summation. We will show that $\sum_{\sigma \in X_{i}}(-1)^{\operatorname{inv}(\sigma)} q^{\operatorname{exc}(\sigma)}=(1-q)^{n}$. Let $X_{i}^{+}=\left\{\sigma \in X_{i}: \sigma_{i}=+i\right\}$ and $X_{i}^{-}=X_{i}-X_{i}^{+}$. For each $\sigma \in X_{i}^{+}$, deleting $i$ and then shrinking $\sigma$ on $[i, n]$ to get $\rho$ will clearly give us all $\rho \in \mathfrak{B}_{n-1}$. An identical statement is again true when we get $\rho \in \mathfrak{B}_{n-1}$ from $\sigma \in X_{i}^{-}$by deleting $\sigma_{i}$ and shrinking $\sigma$ from $[i, n]$.

Let $a_{n}^{+}=\sum_{\sigma \in X_{i}^{+}}(-1)^{\operatorname{in} v_{\mathrm{B}}(\sigma)} q^{\operatorname{exc}(\sigma)}$ and $a_{n}^{-}=\sum_{\sigma \in X_{i}^{-}}(-1)^{\operatorname{in} v_{\mathrm{B}}(\sigma)} q^{\operatorname{exc}(\sigma)}$. We will show that $a_{n}^{+}+a_{n}-=(1-q)^{n}$ by induction on $n$. The result is clear when $n=1,2$ and so let $n>2$. Recall that $\rho$ is obtained by shrinking $\sigma$ on $[i, n]$. By Lemma 6 , $\operatorname{inv}_{\mathrm{B}}(\sigma)=\operatorname{inv}_{\mathrm{B}}(\rho)$ and $\operatorname{exc}(\sigma)=\operatorname{exc}(\rho)$. Thus by induction, $a_{n}^{+}=(1-q)^{n-1}$. To evaluate $a_{n}^{-}$, we get by Lemma 7 that $\operatorname{inv}_{\mathrm{B}}(\sigma) \not \equiv \operatorname{inv}_{\mathrm{B}}(\rho)(\bmod 2)$ and $\operatorname{exc}(\sigma)=\operatorname{exc}(\rho)+1$. Thus by induction, $a_{n}^{-}=-q(1-q)^{n-1}$. Adding the two completes the proof.

We state two corollaries of Theorem 8 when in the cases when $t=1$ and $t=n$ as we only state counterparts of these two when enumeration is done in the symmetric

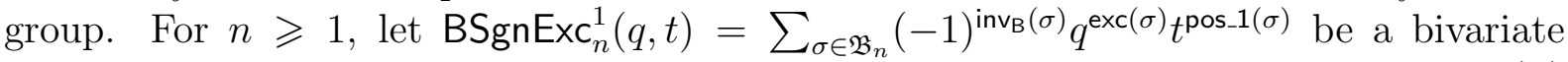
signed enumerator where the exponent of $t$ in the term corresponding to $\sigma$ is pos_1 $(\sigma)$ and let $\operatorname{BSgnExc}_{n}^{n}(q, t)=\sum_{\sigma \in \mathfrak{B}_{n}}(-1)^{\operatorname{inv}_{\mathrm{B}}(\sigma)} q^{\operatorname{exc}(\sigma)} t^{\text {pos_n }(\sigma)}$ be a bivariate signed enumerator where the exponent of $t$ in the term corresponding to $\sigma$ is pos_n $(\sigma)$.

Corollary 9 For $n \geqslant 1$, $\operatorname{BSgnExc}_{n}^{1}(q, t)=t(1-q)^{n}$ and $\operatorname{BSgnExc}_{n}^{n}(q, t)=t^{n}(1-q)^{n}$.

Bivariate enumerators as in Corollary 9 are not known in $\mathfrak{S}_{n}$. This motivates us to define a bivariate signed excedance enumerator for the permutation group $\mathfrak{S}_{n}$. Towards finding such bivariate analogues of Theorem 1, we consider the following $n \times n$ matrices

$$
M_{n}=\left(\begin{array}{ccccc}
1 & q & q & \cdots & q t \\
1 & 1 & q & \cdots & q t^{2} \\
\vdots & \vdots & \vdots & \ddots & \vdots \\
1 & 1 & 1 & \cdots & t^{n}
\end{array}\right) \text { and } M_{n}^{1}=\left(\begin{array}{ccccc}
t & q & q & \cdots & q \\
t^{2} & 1 & q & \cdots & q \\
\vdots & \vdots & \vdots & \ddots & \vdots \\
t^{n} & 1 & 1 & \cdots & 1
\end{array}\right)
$$


i.e. if $M_{n}=\left(m_{i, j}\right)_{1 \leqslant i, j \leqslant n}$, then $m_{i, j}$ is obtained by the following procedure: set $m_{i, j}=q$ if $i<j$, and $m_{i, j}=1$ otherwise. After this, if $j=n$, set $m_{i, j}=m_{i, j} \cdot t^{i}$. Likewise, if $M_{n}^{1}=\left(m_{i, j}^{\prime}\right)$, then, set $m_{i, j}^{\prime}=q$ if $i<j$ and $m_{i, j}^{\prime}=1$ otherwise. After this, if $j=1$, set $m_{i, j}^{\prime}=m_{i, j}^{\prime} \cdot t^{i}$.

For $n \geqslant 1$, define $\operatorname{SgnExc}_{n}^{n}(q, t)=\sum_{\pi \in \mathfrak{S}_{n}}(-1)^{\operatorname{inv}_{\mathrm{A}}(\pi)} q^{\operatorname{exc}(\pi)} t^{\text {pos_n }(\pi)}$. Thus, $\operatorname{SgnExc}_{1}^{1}(q, t)=$ $t$. We get the following bivariate generalization of Theorem 1 .

Theorem 10 For $n \geqslant 2$, $\operatorname{SgnExc}_{n}^{n}(q, t)=t^{n-1}(1-q)^{n-2}(t-q)$.

Proof: Our proof is similar to the proof of Theorem 1 of [5]. Consider the matrix $M_{n}$. Clearly, $\operatorname{det}\left(M_{n}\right)=\sum_{\pi \in \mathfrak{S}_{n}}(-1)^{\operatorname{inv}_{\mathbf{A}}(\pi)} \prod_{i=1}^{n} m_{i, \pi_{i}}$. For a $\pi \in \mathfrak{S}_{n}$, let $T_{\pi}=\prod_{i=1}^{n} m_{i, \pi_{i}}$ be up to sign, the term corresponding to $\pi$ occuring in the determinant expansion. Since $m_{i, j}=q$ if $i<j$ and $m_{i, j}=1$ otherwise, and since $m_{i, n}$ also contributes a factor of $t^{i}$, we get $T_{\pi}=q^{\operatorname{exc}(\pi)} t^{\operatorname{pos} \_n(\pi)}$. Thus, $\operatorname{SgnExc}_{n}(q, t)=\operatorname{det}\left(M_{n}\right)$. Throughout this work, columns of $n \times n$ matrices are numbered from $1 \ldots n$ and $\mathrm{Col}_{i}$ refers to the $i$-th column. We induct on $n$ to show the result. The case when $n=2$ is easy to check and thus assume $n \geqslant 3$. By performing the elementary column operation $\mathrm{Col}_{1}:=\mathrm{Col}_{1}-\mathrm{Col}_{2}$ and then evaluating $\operatorname{det}\left(M_{n}\right)$, it is easy to see that $\operatorname{det}\left(M_{n}\right)=t^{n-1}(1-q)^{n-2}(t-q)$, completing the proof.

For $n \geqslant 1$, define $\operatorname{SgnExc}_{n}^{1}(q, t)=\sum_{\pi \in \mathfrak{S}_{n}}(-1)^{\operatorname{inv}_{A}(\pi)} q^{\operatorname{exc}(\pi)} t^{\text {pos_1 } 1(\pi)}$. We get the following different generalization of Theorem 1 .

Theorem 11 For $n \geqslant 2, \operatorname{SgnExc}_{n}^{1}(q, t)=t(1-q)^{n-2}\left(1-q t^{n-1}\right)$.

Proof: Arguing as in the proof Theorem 10, we get $\operatorname{SgnExc}_{n}^{1}(q, t)=\operatorname{det}\left(M_{n}^{1}\right)$. Performing the elementary row operation $\operatorname{Row}_{1}:=\operatorname{Row}_{1}-$ Row $_{2}$, and then evaluating the determinant makes it easy to see that $\operatorname{det}\left(M_{n}^{1}\right)=t(1-q)^{n-2}\left(1-q t^{n-1}\right)$, completing the proof.

\subsection{Adding quantities to the sign}

In this subsection, we enumerate similar signed excedance statistics. A difference here is that the exponent of -1 is a sum of several statistics of $\sigma$. For $n \geqslant 1$ and $1 \leqslant i \leqslant n$, define $\operatorname{BSgnWkSkExc}_{n}^{i}(q, t)=\sum_{\sigma \in \mathfrak{B}_{n}}(-1)^{\operatorname{inv}_{\mathrm{B}}(\sigma)+\operatorname{wknexc}(\sigma)} q^{\operatorname{exc}(\sigma)} t^{\text {pos_i }(\sigma)}$ as the bivariate weak skew signed excedance enumerator with the exponent of $t$ being the index in $\sigma$ where $i$ up to sign occurs.

Theorem 12 For $n \geqslant 1$ and $1 \leqslant i \leqslant n$, $\operatorname{BSgnWkSkExc}_{n}^{i}(q, t)=t^{i}(-1)^{n}(1+q)^{n}$.

Proof: This proof is very similar to the proof of Theorem 8. Let $\sigma=\left(\sigma_{1}, \sigma_{2}, \ldots, \sigma_{n}\right) \in$ $\mathfrak{B}_{n}$ and as before, let $X_{i}=\left\{\sigma \in \mathfrak{B}_{n}\right.$ : pos_i $\left.(\sigma)=i\right\}$. Consider the map $\tau_{k}:\left(\mathfrak{B}_{n}-X_{i}\right) \mapsto$ $\left(\mathfrak{B}_{n}-X_{i}\right)$ defined as in the proof of Theorem 8 (i.e. change the sign of the smallest element in absolute value in the cycle containing $i)$. Let $\psi=\tau_{k}(\sigma)$. Clearly, pos_i $(\sigma)=$ pos_i $(\psi)$ and further, by Lemma 5 , wknexc $(\sigma)=\operatorname{wknexc}(\psi)$. Thus, as before, we get

$$
\sum_{\sigma \in \mathfrak{B}_{n}-X_{i}}(-1)^{\operatorname{inv}_{\mathrm{B}}(\sigma)+\text { wknexc }(\sigma)} q^{\operatorname{exc}(\sigma)} t^{\text {pos_i }(\sigma)}=0 .
$$


Hence,

$$
\operatorname{BSgnSkExc}_{n}(q, t)=\sum_{\sigma \in X_{i}}(-1)^{\operatorname{inv}_{\mathrm{B}}(\sigma)+\text { whnexc }(\sigma)} q^{\operatorname{exc}(\sigma)} t^{\text {pos_i }(\sigma)}
$$

and thus, a factor of $t^{i}$ can be removed from all terms. We will show that if $a_{n}=$ $\sum_{\sigma \in X_{i}}(-1)^{\operatorname{inv}_{\mathrm{B}}(\sigma)+\text { wknexc }(\sigma)} q^{\operatorname{exc}(\sigma)}$, then $a_{n}=(-1)^{n}(1+q)^{n}$. Again, we induct on $n$ with the base case when $n=1,2$ being easy. Thus, assume $n>2$ and define $X_{i}^{+}=\left\{\sigma \in X_{i}: \sigma_{i}=\right.$ $+i\}$ and $X_{i}^{-}=X_{i}-X_{i}^{+}$.

Let $a_{n}^{+}=\sum_{\sigma \in X_{i}^{+}}(-1)^{\operatorname{inv}(\sigma)+\operatorname{wnnexc}(\sigma)} q^{\operatorname{exc}(\sigma)}$ and $a_{n}^{-}=\sum_{\sigma \in X_{i}^{-}}(-1)^{\operatorname{inv}(\sigma)+\operatorname{wknexc}(\sigma)} q^{\operatorname{exc}(\sigma)}$. We compute $a_{n}^{+}$first. Let $\sigma \in X_{i}^{+}$and let $\rho \in \mathfrak{B}_{n-1}$ be obtained by deleting $i$ and shrinking $\sigma$ on $[i, n]$. By Lemma $6, \operatorname{inv}_{\mathrm{B}}(\sigma)+\operatorname{wknexc}(\sigma) \not \equiv \operatorname{inv}_{\mathrm{B}}(\rho)+\operatorname{wknexc}(\rho)(\bmod 2)$ while $\operatorname{exc}(\sigma)=\operatorname{exc}(\rho)$. Thus, by induction, $a_{n}^{+}=-(1+q)^{n-1}$.

We next compute $a_{n}^{-}$. Let $\sigma \in X_{i}^{-}$and $\rho$ be obtained by deleting $\sigma_{i}$ and shrinking $\sigma$ on $[i, n]$. By Lemma $7, \operatorname{inv}_{\mathrm{B}}(\sigma)+\operatorname{wknexc}(\sigma) \not \equiv \operatorname{inv}_{\mathrm{B}}(\rho)+\operatorname{wknexc}(\rho)(\bmod 2)$ while $\operatorname{exc}(\sigma)=\operatorname{exc}(\rho)+1$. By induction, $a_{n}^{-}=-q \cdot(1+q)^{n-1}$. Adding $a_{n}^{+}$and $a_{n}^{-}$completes the proof.

We define similar bivariate generating functions for the permutation group $\mathfrak{S}_{n}$. For $n \geqslant 1$, define $\operatorname{SgnWkSkExc}_{n}^{n}(q, t)=\sum_{\pi \in \mathfrak{S}_{n}}(-1)^{\operatorname{inv}_{A}(\pi)+\operatorname{wknexc}(\pi)} q^{\operatorname{exc}(\pi)} t^{\text {pos_n }(\pi)}$. Likewise, for $n \geqslant 1$, define $\operatorname{SgnWkSkExc}_{n}^{1}(q, t)=\sum_{\pi \in \mathfrak{S}_{n}}(-1)^{\operatorname{inv}_{\mathrm{A}}(\pi)+\text { wknexc }(\pi)} q^{\operatorname{exc}(\pi)} t^{\text {pos_1 } 1(\pi)}$. Consider the following $n \times n$ matrices.

$$
T_{n}=\left(\begin{array}{ccccc}
-1 & q & q & \cdots & q t \\
-1 & -1 & q & \cdots & q t^{2} \\
\vdots & \vdots & \vdots & \ddots & \vdots \\
-1 & -1 & -1 & \cdots & -t^{n}
\end{array}\right) \text { and } T_{n}^{1}=\left(\begin{array}{ccccc}
-t & q & q & \cdots & q \\
-t^{2} & -1 & q & \cdots & q \\
\vdots & \vdots & \vdots & \ddots & \vdots \\
-t^{n} & -1 & -1 & \cdots & -1
\end{array}\right)
$$

If $T_{n}=\left(t_{i, j}\right)_{1 \leqslant i, j \leqslant n}$, then $t_{i, j}$ is obtained as follows: set $t_{i, j}=q$ if $i<j$, and $t_{i, j}=-1$ otherwise. Then, if $j=n$, set $t_{i, j}=t_{i, j} \cdot t^{i}$. Similarly, if $T_{n}^{1}=\left(t_{i, j}^{\prime}\right)_{1 \leqslant i, j \leqslant n}$, then $t_{i, j}^{\prime}$ obtained as follows: set $t_{i, j}^{\prime}=q$ if $i<j$, and $t_{i, j}^{\prime}=-1$ otherwise. Then, if $j=1$, set $t_{i, j}=t_{i, j} \cdot t^{i}$. We get the following generalizations of Theorem 2 of [5].

Theorem 13 For $n \geqslant 2$, SgnWkSkExc ${ }_{n}^{n}(q, t)=(-1)^{n} t^{n-1}(1+q)^{n-2}(t+q)$. Similarly, for $n \geqslant 2, \operatorname{SgnWkSkExc}_{n}^{1}(q, t)=(-1)^{n} t(1+q)^{n-2}\left(1+q t^{n-1}\right)$.

Proof: Arguing as in the proof of Theorem 10, we get $\operatorname{SgnWkSkExc}_{n}^{n}(q, t)=\operatorname{det}\left(T_{n}\right)$. Performing the elementary column operation $\mathrm{Col}_{1}:=\mathrm{Col}_{1}-\mathrm{Col}_{2}$ and then evaluating $\operatorname{det}\left(T_{n}\right)$, it can be checked that $\operatorname{det}\left(T_{n}\right)=(-1)^{n} t^{n-1}(1+q)^{n-2}(t+q)$. Similarly, it is easy to see that $\operatorname{SgnWkSkExc}_{n}^{1}(q, t)=\operatorname{det}\left(T_{n}^{1}\right)$. Evaluating the determinant after performing the column operation $\mathrm{Col}_{n}:=\mathrm{Col}_{n}-\mathrm{Col}_{n-1}$ shows that $\operatorname{det}\left(T_{n}^{1}\right)=(-1)^{n} t(1+q)^{n-2}\left(1+q t^{n-1}\right)$, completing the proof.

We next move on to type-B analogues of Theorem 5 of [5]. For $n \geqslant 1$ and for $1 \leqslant i \leqslant n$, let $\mathrm{BSgnSkExc}_{n}^{i}(q, t)=\sum_{\sigma \in \mathfrak{B}_{n}}(-1)^{\operatorname{inv}_{\mathrm{B}}(\sigma)+\operatorname{nexc}(\pi)} q^{\operatorname{exc}(\sigma)} t^{\text {pos_i }(\sigma)}$ be a bivariate skew signed enumerator counting excedance and the index where $i$ up to sign occurs. 
Theorem 14 For $n \geqslant 1$ and $1 \leqslant i \leqslant n, \operatorname{BSgnSkExc}_{n}^{i}(q, t)=t^{i}(1-q)^{n}$.

Proof: This proof is very similar to the proof of Theorem 12, and hence we just mention the differences. As before, define $X_{i}=\left\{\sigma \in \mathfrak{B}_{n}\right.$ : pos_i $\left.(\sigma)=i\right\}$. It is easy to show that $\sum_{\sigma \in \mathfrak{B}_{n}-X_{i}}(-1)^{\operatorname{inv}_{\mathrm{B}}(\sigma)+\operatorname{nexc}(\sigma)} q^{\operatorname{exc}(\sigma)} t^{\text {pos_i }(\sigma)}=0$.

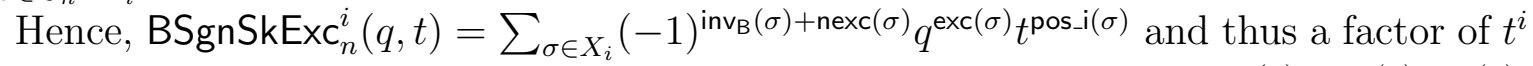
can be removed from all terms. We will show that if $a_{n}=\sum_{\sigma \in X_{i}}(-1)^{\operatorname{inv}(\sigma)+\operatorname{nexc}(\sigma)} q^{\operatorname{exc}(\sigma)}$, then $a_{n}=(1-q)^{n}$. Again, define $X_{i}^{+}=\left\{\sigma \in X_{i}: \sigma_{i}=+i\right\}$ and $X_{i}^{-}=X_{i}-X_{i}^{+}$. Let $a_{n}^{+}=\sum_{\sigma \in X_{i}^{+}}(-1)^{\operatorname{inv}(\sigma)+\operatorname{nexc}(\sigma)} q^{\operatorname{exc}(\sigma)}$ and let $a_{n}^{-}=\sum_{\sigma \in X_{i}^{-}}(-1)^{\operatorname{inv}(\sigma)+\operatorname{nexc}(\sigma)} q^{\operatorname{exc}(\sigma)}$.

Let $\sigma \in \mathfrak{B}_{n}$ and $\rho$ be obtained by deleting $i$ and by shrinking $\sigma$ on $[i, n]$. By induction and Lemma 6, as $\operatorname{inv}_{\mathrm{B}}(\sigma)+\operatorname{nexc}(\sigma)=\operatorname{inv}_{\mathrm{B}}(\rho)+\operatorname{nexc}(\rho)$ and as $\operatorname{exc}(\sigma)=\operatorname{exc}(\rho)$, we get $a_{n}^{+}=a_{n-1}$. Similarly, using Lemma 7 , we get $a_{n}^{-}=-q a_{n-1}$. Adding the two completes the proof.

We consider bivariate analogues of Theorem 14 when enumeration is done in $\mathfrak{S}_{n}$. For $n \geqslant 1$, let $\operatorname{SgnSkExc}_{n}(q, t)=\sum_{\pi \in \mathfrak{S}_{n}}(-1)^{\operatorname{inv}_{A}(\pi)+\operatorname{nexc}(\pi)} q^{\operatorname{exc}(\pi)} t^{\text {pos_n }(\pi)}$ be its bivariate signed skew excedance enumerator and let $\operatorname{SgnSkExc}_{0}(q, t)=1$. Consider the following $n \times n$ matrices

$$
U_{n}=\left(\begin{array}{ccccc}
1 & q & q & \cdots & q t \\
-1 & 1 & q & \cdots & q t^{2} \\
\vdots & \vdots & \vdots & \ddots & \vdots \\
-1 & -1 & -1 & \cdots & t^{n}
\end{array}\right)
$$

i.e. if $U_{n}=\left(u_{i, j}\right)_{1 \leqslant i, j \leqslant n}$, then $u_{i, j}$ is obtained as follows: set $u_{i, j}=q$ if $i<j, u_{i, j}=1$ if $i=j$ and $u_{i, j}=-1$ otherwise. After this, if $j=n$, set $u_{i, j}=u_{i, j} \cdot t^{i}$.

We give the following bivariate analogue of Theorem 5 of [5]. First consider the sequence of bivariate polynomials defined by the the following recurrence:

$$
d_{n}(q, t)=(3-q) t \cdot d_{n-1}(q, t)+2 t^{2}(q-1) \cdot d_{n-2}(q, t)+2^{n-3} q t(1-t) .
$$

with $d_{0}(q, t)=1$ and $d_{1}(q, t)=t$. A few initial $d_{n}(q, t)$ 's are given below.

$$
\begin{aligned}
d_{0}(q, t)= & 1 \\
d_{1}(q, t)= & t \\
d_{2}(q, t)= & t^{2}+t q \\
d_{3}(q, t)= & t^{3}+q\left(t^{3}+t^{2}+2 t\right)-q^{2} t^{2} \\
d_{4}(q, t)= & t^{4}+q\left(4 t^{4}+t^{3}+2 t^{2}+4 t\right)-q^{2}\left(t^{4}+2 t^{3}+2 t^{2}\right)+q^{3} t^{3} \\
d_{5}(q, t)= & t^{5}+q\left(11 t^{5}+t^{4}+2 t^{3}+4 t^{2}+8 t\right)-q^{2}\left(5 t^{5}-3 t^{4}-4 t^{3}-4 t^{2}\right) \\
& +q^{3}\left(t^{5}+3 t^{4}+2 t^{3}\right)-q^{4} t^{4}
\end{aligned}
$$

Theorem 15 For $n \geqslant 0$, $\operatorname{SgnSkExc}_{n}(q, t)=d_{n}(q, t)$. 
Proof: Let $U_{n}$ be the matrix defined above. By using arguments as done before, we see that $\operatorname{det}\left(U_{n}\right)=\operatorname{SgnSkExc}_{n}(q, t)$. We claim that $\operatorname{det}\left(U_{n}\right)=d_{n}(q, t)$. By performing the elementary column operation $\mathrm{Col}_{1}:=\mathrm{Col}_{1}-\mathrm{Col}_{2}$ and then evaluating $\operatorname{det} U_{n}$, we get the following recurrence. We note that on setting $t=1$, we recover the recurrence given in the proof of Theorem 5 of [5].

$$
\operatorname{det} U_{n}=(3-q) t \cdot \operatorname{det} U_{n-1}+2 t^{2}(q-1) \cdot \operatorname{det} U_{n-2}+2^{n-3} q t(1-t) .
$$

where $\operatorname{det} U_{0}=1, \operatorname{det} U_{1}=t$ and $\operatorname{det} U_{2}=t^{2}+t q$ are easy to observe. It is clear that recurrence (3) is identical to the recurrence (2) which completes this proof.

Remark 16 The coefficient of $q$ in $d_{n}(q, 1)$ is the Eulerian number $A_{n, 2}$ (see the proof of Theorem 5 of [5]). In the bivariate analogue obtained, when $t$ is a variable, the coefficient of $q$, gives a polynomial analogue of $A_{n, 2}$ in the variable $t$. The Online Encyclopedia of Integer Sequences (see sequence id:A054123) mentions right Fibonacci row-sum arrays where this polynomial refinement of $A_{n, 2}$ occurs in even rows. This sequence thus has occurred while enumerating a different problem and appears again here. We do not know any connection between the two problems.

\subsection{Adding quantities to $q$ 's exponent as well}

In this subsection, our generating function has a slightly modified exponent of $q$. For $n \geqslant 1$, and for $1 \leqslant i \leqslant n$, define

$$
\operatorname{BSgnWkSkWkExc}_{n}^{i}(q, t)=\sum_{\sigma \in \mathfrak{B}_{n}}(-1)^{\operatorname{inv}_{\mathrm{B}}(\sigma)+\text { wknexc }(\sigma)} q^{\text {wkexc }(\sigma)} t^{\text {pos_i }(\sigma)} .
$$

Thus the fixed points of $\sigma$ contribute to the exponents of both -1 and $q$.

Theorem 17 For $n \geqslant 1$ and $1 \leqslant i \leqslant n$, $\operatorname{BSgnWkSkWkExc}_{n}^{i}(q, t)=t^{i}(-2 q)^{n}$.

Proof: This proof is very similar to earlier proofs and thus, we just mention the differences. As before, we get $\sum_{\sigma \in \mathfrak{B}_{n}-X_{i}}(-1)^{\operatorname{inv}(\sigma)+\operatorname{wknexc}(\sigma)} q^{\text {wkexc }(\sigma)} t^{\text {pos } i(\sigma)}=0$ where $X_{i}=$ $\left\{\sigma \in \mathfrak{B}_{n}:\right.$ pos_i $\left.(\sigma)=i\right\}$.

Hence BSgnWkSkWkExc $n(q, t)=\sum_{\sigma \in X_{i}}(-1)^{\operatorname{inv}_{B}(\sigma)+\text { wknexc }(\sigma)} q^{\text {wkexc }(\sigma)} t^{\text {posi } i(\sigma)}$ and thus a factor of $t^{i}$ can be removed from all terms. If $a_{n}=\sum_{\sigma \in X_{i}}(-1)^{\operatorname{inv_{B}}(\sigma)+\operatorname{wknexc}(\sigma)} q^{\operatorname{wkexc}(\sigma)}$, then we need to show that $a_{n}=(-2 q)^{n}$. We do this by induction on $n$. The base case when $n=1,2$ are easy. Thus, assume $n>2$ and as in earlier proofs, define $X_{n}^{+}$and $X_{n}^{-}$. Let $a_{n}^{+}=\sum_{\sigma \in X_{i}^{+}}(-1)^{\operatorname{inv}(\sigma)+\operatorname{wknexc}(\sigma)} q^{\text {wkexc }(\sigma)}$ and let $a_{n}^{-}=\sum_{\sigma \in X_{i}^{-}}(-1)^{\operatorname{inv}(\sigma)+\operatorname{wknexc}(\sigma)} q^{\text {wkexc }(\sigma)}$.

For $\sigma \in X_{i}^{+}$, define $\rho \in \mathfrak{B}_{n-1}$ by deleting $i$ and shrinking $\sigma$ on $[i, n]$. By Lemma 6 as $\operatorname{inv}_{\mathrm{B}}(\sigma)+\operatorname{wknexc}(\sigma) \not \equiv \operatorname{inv}_{\mathrm{B}}(\rho)+\operatorname{wknexc}(\rho)$ and as the fixed point $i$ contributes 1 to the exponent of $q$, we get $a_{n}^{+}=-q\left(a_{n-1}\right)$. For $\sigma \in X_{i}^{-}$, define $\rho \in \mathfrak{B}_{n-1}$ by deleting $\bar{i}$

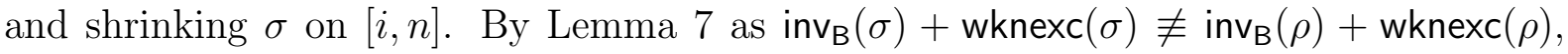
we get a negative sign, and as $\sigma_{i}=\bar{i}$, we get $\operatorname{wkexc}(\sigma)=\operatorname{wk\operatorname {exc}}(\rho)+1$ and so we get $a_{n}^{-}=-q\left(a_{n-1}\right)$. Adding $a_{n}^{+}$and $a_{n}^{-}$completes the proof. 
We derive similar bivariate results when enumeration is done in the symmetric group $\mathfrak{S}_{n}$. Define SgnWkSkWkExc ${ }_{n}(q, t)=\sum_{\pi \in \mathfrak{S}_{n}}(-1)^{\operatorname{inv}_{A}(\pi)+\operatorname{wknexc}(\pi)} q^{\operatorname{wkexc}(\pi)} t^{\text {pos_n }(\pi)}$ as the bivariate signed weak-skew weak-excedance enumerator. Let $\operatorname{SgnWkSkWkExc}_{0}(q, t)=1$. Consider the following $n \times n$ matrix.

$$
F_{n}=\left(\begin{array}{ccccc}
-q & q & q & \cdots & q t \\
-1 & -q & q & \cdots & q t^{2} \\
\vdots & \vdots & \vdots & \ddots & \vdots \\
-1 & -1 & -1 & \cdots & -q t^{n}
\end{array}\right)
$$

Likewise if $F_{n}=\left(f_{i, j}\right)_{1 \leqslant i, j \leqslant n}$, then $f_{i, j}$ is obtained as follows: set $f_{i, j}=q$ if $i<j$, $f_{i, j}=-q$ if $i=j$ and $f_{i, j}=-1$ otherwise. Then, if $j=n$, set $f_{i, j}=f_{i, j} \cdot t^{i}$. Consider the sequence of bivariate polynomials defined as follows:

$$
f_{n}=(1-3 q) t \cdot f_{n-1}+2 q t^{2}(q-1) f_{n-2}+(-1)^{n}(q-1)^{n-2}\left(q t-q t^{2}\right)
$$

with $f_{0}(q, t)=1$ and $f_{1}(q, t)=-t q$. We tabulate $f_{n}(q, t)$ for a few values of $n$ below.

$$
\begin{aligned}
f_{0}(q, t)= & 1 \\
f_{1}(q, t)= & -t q \\
f_{2}(q, t)= & t q+t^{2} q^{2} \\
f_{3}(q, t)= & t q-\left(t^{3}+2 t^{2}+t\right) q^{2}-t^{3} q^{3} \\
f_{4}(q, t)= & t q-\left(t^{4}+2 t^{2}+2 t\right) q^{2}+\left(4 t^{4}+4 t^{3}+2 t^{2}+t\right) q^{3}+t^{4} q^{4} \\
f_{5}(q, t)= & t q-\left(t^{5}+2 t^{2}+3 t\right) q^{2}+\left(5 t^{5}+4 t^{3}+4 t^{2}+3 t\right) q^{3} \\
& -\left(11 t^{4}+8 t^{4}+4 t^{3}++2 t^{2}+t\right) q^{4}-t^{5} q^{5}
\end{aligned}
$$

Theorem 18 For $n \geqslant 0$, BSgnWkSkWkExc ${ }_{0}(q, t)=f_{n}(t, q)$.

Proof: Consider the matrix $F_{n}$ given above. We get $\operatorname{BSgnWkSkWkExc}(q, t)=\operatorname{det}\left(F_{n}\right)$ by arguing as done earlier. We claim that $\operatorname{det}\left(F_{n}\right)=f_{n}(q, t)$. Performing the column operation $\mathrm{Col}_{1}:=\mathrm{Col}_{1}-\mathrm{Col}_{2}$ and then evaluating the determinant gives us the following recurrence:

$$
\operatorname{det} F_{n}=(1-3 q) t \cdot \operatorname{det} F_{n-1}+2 q t^{2}(q-1) \operatorname{det} F_{n-2}+(-1)^{n}(q-1)^{n-2}\left(q t-q t^{2}\right)
$$

We note that recurrence (5) is identical to recurrence (4). Since the initial values of $f_{n}(q, t)$ and $\operatorname{det} F_{n}$ are identical and they satisfy the same recurrence, they are identical for all $n$, completing the proof. 


\section{Enumerating excedance like statistics}

For a $\sigma \in \mathfrak{B}_{n}$, define its excedance-sum as excsum $(\sigma)=\sum_{i \in \operatorname{ExcSet}(\sigma)} i$. For the next result which is an analogue of Theorem 8 of [5], we recall the following notation used in $q$-series theory. Let $q$ be a variable and for a non negative integer $n$, define

$$
(q ; q)_{n}= \begin{cases}1 & \text { if } n=0 \\ \prod_{i=1}^{n}\left(1-q^{i}\right) & \text { if } n>0\end{cases}
$$

For a non-negative integer $i$, we also recall that $[i]_{q}=1+q+q^{2}+\cdots+q^{i-1}$, and that $[0]_{q}=0$. For $n \geqslant 1$ and $1 \leqslant i \leqslant n$, define the bivariate signed excedance-sum enumerator to be $\operatorname{BSgnExcSum}_{n}^{i}(q)=\sum_{\sigma \in \mathfrak{B}_{n}}(-1)^{\operatorname{inv}(\sigma)} q^{\text {excsum }(\sigma)} t^{\text {pos } \mathrm{i}(\sigma)}$.

Theorem 19 For $n \geqslant 1$ and $1 \leqslant i \leqslant n, \operatorname{BSgnExcSum}_{n}^{i}(q)=t^{i} \cdot(q ; q)_{n}$.

Proof: Let $X_{i}=\left\{\sigma \in \mathfrak{B}_{n}\right.$ : pos_i $\left.(\sigma)=i\right\}$. For $\sigma \in \mathfrak{B}_{n}-X_{i}$, the cycle $C$ containing $i$ has length at least two. Let $\tau_{k}:\left(\mathfrak{B}_{n}-X_{i}\right) \mapsto\left(\mathfrak{B}_{n}-X_{i}\right)$ be the map which flips the sign of the minimum element of $C$ in absolute value. Let $\psi=\tau_{k}(\sigma)$. Hence, for all indices $i \neq k$, we have $\sigma_{i}=\psi_{i}$ while we have $\sigma_{k}=i=-\psi_{k}$. Lemma 5 shows that $\operatorname{ExcSet}(\sigma)=$ $\operatorname{ExcSet}(\psi)$ which implies that $\operatorname{excsum}(\sigma)=\operatorname{excsum}(\psi)$. Further, pos_i $(\sigma)=\operatorname{pos}_{-} i(\psi)$ and $\operatorname{inv}_{\mathrm{B}}(\sigma) \not \equiv \operatorname{inv}_{\mathrm{B}}(\psi)(\bmod 2)$ by Lemma 3. Thus, $\sum_{\sigma \in \mathfrak{B}_{n}-X_{i}}(-1)^{\operatorname{inv}_{\mathrm{B}}(\sigma)} q^{\text {excsum }(\sigma)} t^{\text {pos_i }(\sigma)}=0$.

Hence, only elements of $X_{i}$ contribute to the sum concerned. Since each $\sigma \in X_{i}$ has pos_i $\left(\sigma_{i}\right)=i$, we can pull out the term $t^{i}$ from the sum. Actually, the above argument shows that whenever some $\sigma \in \mathfrak{B}_{n}$ has a cycle $C$ of length at least two, we can negate its minimum element in absolute value to get $\psi$ and cancel out terms arising from $\sigma$ by assigning the $2^{n}$ signs to $\sigma$ (as $\sigma$ and $\psi$ will have opposite parity but have $\operatorname{ExcSet}(\sigma)=$ $\operatorname{ExcSet}(\psi))$.

Thus, the only terms that survive the cancellations come from assigning signs to the identity permutation $e=1,2, \ldots, n$. We are left with finding the signed excedance-sum over the $2^{n}$ signed permutations of $e$. Applying Corollary 4 to the identity permutation, it is easy to see that $\operatorname{inv}_{\mathrm{B}}(\sigma)$ has the same parity as the number of negative entries in $\sigma$ and $\operatorname{excsum}(\sigma)$ is the sum of the negative entries in $\sigma$. Thus, we get $\operatorname{BSgnExcSum}_{n}^{i}(q)=$ $\sum_{S \subseteq[n]}(-1)^{|S|} q^{\sum_{x \in S} x}=\prod_{i=1}^{n}\left(1-q^{i}\right)=(q ; q)_{n}$. Multiplication by $t^{i}$ completes the proof.

We give a bivariate analogue of Theorem 8 of [5] below. Consider the following $n \times n$ matrices

$$
P_{n}=\left(\begin{array}{ccccc}
1 & q & q & \cdots & q t \\
1 & 1 & q^{2} & \cdots & (q t)^{2} \\
\vdots & \vdots & \vdots & \ddots & \vdots \\
1 & 1 & 1 & \cdots & (q t)^{n-1} \\
1 & 1 & 1 & \cdots & t^{n}
\end{array}\right) \text { and } S_{n}=\left(\begin{array}{ccccc}
1 & q & q^{2} & \cdots & q^{n-1} t \\
1 & 1 & q & \cdots & q^{n-2} t^{2} \\
\vdots & \vdots & \vdots & \ddots & \vdots \\
1 & 1 & 1 & \cdots & q t^{n-1} \\
1 & 1 & 1 & \cdots & t^{n}
\end{array}\right)
$$

i.e. if $P_{n}=\left(p_{i, j}\right)_{1 \leqslant i, j \leqslant n}$, then $p_{i, j}$ is obtained by the following procedure: set $p_{i, j}=q^{i}$ if $i<j$ and $p_{i, j}=1$ otherwise. Then, set $p_{i, j}=p_{i, j} \cdot t^{i}$ if $j=n$. Similarly, if $S_{n}=\left(s_{i, j}\right)_{1 \leqslant i, j \leqslant n}$, 
then $s_{i, j}$ is obtained as follows: set $s_{i, j}=q^{j-i}$ if $i<j$ and $s_{i, j}=1$ otherwise. After this, set $s_{i, j}=s_{i, j} \cdot t^{i}$ if $j=n$.

For a $\pi \in \mathfrak{S}_{n}$, define its excedance set as $\operatorname{ExcSet}(\pi)=\left\{i \in[n]: \pi_{i}>i\right\}$. Define its excedance-sum as excsum $(\pi)=\sum_{i \in \operatorname{ExcSet}(\pi)} i$ and its excedance-length as $\operatorname{ExcLen}(\pi)=$ $\sum_{i \in \operatorname{ExcSet}(\pi)}\left(\pi_{i}-i\right)$. For $n \geqslant 1$, let $\operatorname{SgnExcSum}_{n}(q, t)=\sum_{\pi \in \mathfrak{S}_{n}}(-1)^{\operatorname{inv} v_{A}(\pi)} q^{\operatorname{excsum}(\pi)} t^{\text {pos_n }(\pi)}$ to be the bivariate signed excedance-sum enumerator. Similarly, define $\operatorname{SgnExcLen}_{n}(q, t)=$ $\sum_{\pi \in \mathfrak{S}_{n}}(-1)^{\operatorname{inv}_{A}(\pi)} q^{\operatorname{ExcLen}(\pi)} t^{\text {pos_n }(\pi)}$ as the bivariate signed excedance-length enumerator.

Theorem 20 For integers $n \geqslant 1$, $\operatorname{SgnExcSum}_{n}(q, t)=t^{n-1}\left(t-q^{n-1}\right) \prod_{i=1}^{n-2}\left(1-q^{i}\right)$.

Proof: It is clear that $\operatorname{SgnExcSum}_{n}(q, t)=\operatorname{det}\left(P_{n}\right)$. Let $\operatorname{Row}_{i}$ denote the $i$-th row of any $n \times n$ matrix for $1 \leqslant i \leqslant n$ (the matrix will be clear from the context). Perform the elementary row operation $\operatorname{Row}_{n}:=\operatorname{Row}_{n}-\operatorname{Row}_{n-1}$ and then evaluate the determinant to see that $\operatorname{det}\left(P_{n}\right)=t^{n-1}\left(t-q^{n-1}\right) \prod_{i=1}^{n-2}\left(1-q^{i}\right)$, completing the proof.

Theorem 21 For integers $n \geqslant 1$, $\operatorname{SgnExcLen}_{n}(q, t)=t^{n-1}(t-q)(1-q)^{n-2}$.

Proof: It is clear that $\operatorname{SgnExcLen}_{n}(q, t)=\operatorname{det}\left(S_{n}\right)$ and performing as before the operation $\operatorname{Row}_{n}:=\operatorname{Row}_{n}-\operatorname{Row}_{n-1}$ and then evaluating the determinant gives us $\operatorname{det}\left(S_{n}\right)=$ $t^{n-1}(t-q)(1-q)^{n-2}$, completing the proof.

\section{Signed excedance enumeration in $\mathfrak{B} \mathfrak{D}_{n}$}

Let $\mathfrak{B} \mathfrak{D}_{n}$ be the set of derangements of $\mathfrak{B}_{n} . \mathfrak{B D}_{n}$ consists of the $\sigma \in \mathfrak{B}_{n}$ such that $\sigma_{i} \neq i$ for all $i \in[n]$. For $n \geqslant 1$ and $1 \leqslant i \leqslant n$, define

$$
\operatorname{BDSgnExc}_{n}^{i}(q, t)=\sum_{\sigma \in \mathfrak{B D}_{n}}(-1)^{\operatorname{inv}_{\mathrm{B}}(\sigma)} q^{\operatorname{exc}(\sigma)} t^{\text {pos_i }(\sigma)} .
$$

Define $\operatorname{BDSgnExc}_{0}^{0}(q, t)=1$ and $\operatorname{BDSgnExc}_{0}^{1}(q, t)=t$.

Theorem 22 For $n \geqslant 1$ and $1 \leqslant i \leqslant n, \operatorname{BDSgnExc}_{n}^{i}(q, t)=t^{i}(-q)^{n}$.

Proof: This proof is very similar to that of Theorem 8 and thus we only mention the differences. Since $\sigma$ is a derangement, for all $1 \leqslant i \leqslant n, \sigma_{i} \neq i$. As before, let $X_{i}=\left\{\sigma \in \mathfrak{B}_{n}: \sigma_{i}=-i\right\}$. Since for all $\sigma \in \mathfrak{B}_{n}-X_{i}$ the element $i$ is in a cycle $C$ of length at least two, consider the involution on $\mathfrak{B}_{n}-X_{i}$ defined by changing the sign of the minimum element in absolute value of $C$. Let $\psi$ be the resulting signed permutation. Clearly, $\psi \in \mathfrak{B D}_{n}$. By similar arguments, $\sum_{\sigma \in \mathfrak{B}_{n}-X_{i}}(-1)^{\operatorname{inv}_{\mathrm{B}}(\sigma)} t^{\text {pos_i }(\sigma)} q^{\operatorname{exc}(\sigma)}=0$.

Thus, only derangements with $\sigma_{i}=-i$ contribute to the sum. The argument actually shows that if $\sigma \in \mathfrak{B}_{n}$ has a cycle of length at least two, it will get cancelled. Thus we see that the unique derangement which contributes to the sum has $\sigma(i)=-i$ for all $1 \leqslant i \leqslant n$. Since pos_i $(\sigma)=i, \operatorname{exc}(\sigma)=n$ and $\operatorname{inv}_{\mathrm{B}}(\sigma)=n^{2} \equiv n(\bmod 2)$, we have $\operatorname{BDSgnExc}_{n}^{i}(q, t)=t^{i}(-q)^{n}$, completing the proof. 
We record two simple corollaries of Theorem 22 in the cases when $i=1, n$. We will use them in the next subsection. Define $\operatorname{BDSgnExc}_{n}^{n}(q, t)=\sum_{\sigma \in \mathfrak{B D}_{n}}(-1)^{\operatorname{inv}_{\mathrm{B}}(\sigma)} q^{\operatorname{exc}(\sigma)} t^{\text {pos_n }(\sigma)}$ and $\operatorname{BDSgnExc}_{n}^{1}(q, t)=\sum_{\sigma \in \mathfrak{B D}_{n}}(-1)^{\operatorname{inv}_{\mathrm{B}}(\sigma)} q^{\operatorname{exc}(\sigma)} t^{\text {pos_1 } 1(\sigma)}$.

Corollary 23 For $n \geqslant 1$, $\operatorname{BDSgnExc}_{n}^{n}(q, t)=(-q t)^{n}$ and $\operatorname{BDSgnExc}_{n}^{1}(q, t)=t(-q)^{n}$.

We give a similar bivariate generalization of this result to $\mathfrak{S}_{n}$. Consider the following matrices.

$$
D_{M_{n}}=\left(\begin{array}{ccccc}
0 & q & q & \cdots & q t \\
1 & 0 & q & \cdots & q t^{2} \\
\vdots & \vdots & \vdots & \ddots & \vdots \\
1 & 1 & 1 & \cdots & 0
\end{array}\right) \quad D_{M_{n}}^{1}=\left(\begin{array}{ccccc}
0 & q & q & \cdots & q \\
t^{2} & 0 & q & \cdots & q \\
\vdots & \vdots & \vdots & \ddots & \vdots \\
t^{n} & 1 & 1 & \cdots & 0
\end{array}\right)
$$

We obtain $D_{M_{n}}=\left(d_{i, j}\right)_{1 \leqslant i, j \leqslant n}$ by the following procedure. Set $D_{M_{n}}=M_{n}$, where recall that the matrix $M_{n}$ was defined in Section 3. After this, set $d_{i, i}=0$ for all $1 \leqslant i \leqslant n$. Similarly, we get the entries of $D_{M_{n}}^{1}$ from $M_{n}^{1}$ by making a copy and then changing all diagonal elements to zero.

Motivated by the above result, define $\operatorname{DSgnExc}_{n}^{n}(q, t)=\sum_{\pi \in \mathfrak{D}_{n}}(-1)^{\operatorname{inv}_{\mathrm{A}}(\pi)} t^{\text {pos } \_n(\pi)} q^{\operatorname{exc}(\pi)}$ as the bivariate signed excedance enumerator summed over derangements in $\mathfrak{S}_{n}$. Similarly, define DSgnExc ${ }_{n}^{1}(q, t)=\sum_{\pi \in \mathfrak{D}_{n}}(-1)^{\operatorname{inv}_{\mathrm{A}}(\pi)} t^{\text {pos_1 } 1(\pi)} q^{\operatorname{exc}(\pi)}$ as the bivariate signed excedance enumerator with respect to position of 1 , summed over derangements. We recall that for a positive integer $i,[i]_{q t}=1+q t+(q t)^{2}+\cdots+(q t)^{i-1}$. Define $[0]_{q t}=0$. We show the following bivariate generalizations of Theorem 2 .

Theorem 24 For all positive integers $n \geqslant 1, \operatorname{DSgnExc}_{n}^{n}(q, t)=(-1)^{n-1} q t \cdot[n-1]_{q t}$ and similarly, DSgnExc ${ }_{n}^{1}(q, t)=(-1)^{n-1} q t^{2} \cdot[n-1]_{q t}$.

Proof: It is clear that $\operatorname{DSgnExc}_{n}(q, t)=\operatorname{det}\left(D_{M_{n}}\right)$. Perform the row operation $\operatorname{Row}_{n}:=$ Row $_{n}-$ Row $_{n-1}$ and then evaluate the determinant. It is easy by using induction on $n$ to see that $\operatorname{det}\left(D_{M_{n}}\right)=(-1)^{n-1} q t \cdot[n-1]_{q t}$. Similarly, it is clear that $\operatorname{DSgnExc}_{n}^{1}(q, t)=$ $\operatorname{det}\left(D_{M_{n}^{1}}\right)$. Performing the column operation $\mathrm{Col}_{n}:=\mathrm{Col}_{n}-\mathrm{Col}_{n-1}$ and then evaluating the determinant, it is easy to see that $\operatorname{det}\left(D_{M_{n}^{1}}\right)=(-1)^{n-1} q t^{2}[n-1]_{q t}$, completing the proof.

\subsection{A binomial type equation}

Chen, Tang and Zhao in [2] considered the following polynomials $B_{n}(q)=\sum_{\sigma \in \mathfrak{B}_{n}} q^{\operatorname{exc}(\sigma)}$ and $d_{n}^{B}(q)=\sum_{\sigma \in \mathfrak{B D}_{n}} q^{\operatorname{exc}(\sigma)}$, where, they also use Brenti's definition for excedance in $\mathfrak{B}_{n}$. They showed (see Equation (3.6) of [2]) that

$$
B_{n}(q)=\sum_{k=0}^{n}\left(\begin{array}{l}
n \\
k
\end{array}\right) d_{k}^{B}(q)
$$


Recall that $\operatorname{BDSgnExc}_{1}^{1}(q, t)=-q t$ in contrast to the $\mathfrak{S}_{n}$ case where there are no derangements when $n=1$. The following corollary follows immediately from Corollaries 9 and 23. Note that when $t=1$, Corollary 25 is identical to (7).

Corollary $25 \operatorname{BSgnExc}_{n}^{n}(q, t)=\sum_{k=0}^{n}\left(\begin{array}{l}n \\ k\end{array}\right) t^{n-k} \operatorname{BDSgnExc}_{k}^{k}(q, t)$.

Recall $\mathrm{BSgnExc}_{n}^{1}(q, t)$, the bivariate signed excedance enumerator in $\mathfrak{B}_{n}$ defined in Section 3. Our next corollary is again immediate from Corollaries 9 and 23. Corollary 26 is identical to (7).

Corollary $26 \operatorname{BSgnExc}_{n}^{1}(q, t)=\sum_{k=0}^{n}\left(\begin{array}{l}n \\ k\end{array}\right) \operatorname{BDSgnExc}_{k}^{1}(q, t)$.

\subsection{Signed Excedance Sum}

In this last subsection, we give hyperoctahedral analogues of Theorem 19 of [5]. For $n \geqslant 1$ and $1 \leqslant i \leqslant n$, define $\operatorname{BDSgnExc}_{n}^{i}(q, t)=\sum_{\sigma \in \mathfrak{B D}_{n}}(-1)^{\operatorname{inv}_{\mathrm{B}}(\sigma)} q^{\operatorname{excsum}(\sigma)} t^{\text {posi } i(\sigma)}$.

Theorem 27 For $n \geqslant 1$ and $1 \leqslant i \leqslant n, \operatorname{BDSgnExc}_{n}^{i}(q, t)=t^{i}(-1)^{n} q^{\left(\begin{array}{c}n+1 \\ 2\end{array}\right)}$.

Proof: This proof is very similar to the proof of Theorem 24 and thus we only mention the differences. Let $X_{i}=\left\{\sigma \in \mathfrak{B D}_{n}: \sigma_{i}=-i\right\}$. Consider the involution $\tau_{k}$ on $\mathfrak{B D}_{n}-X_{i}$ defined in the proof of Theorem 19 (i.e. changing the sign of the minimum element in absolute value in the cycle containing $i$ ). The rest of the argument is similar and we omit the details. By induction, the unique derangement that contributes has $\sigma(i)=-i$ for all $1 \leqslant i \leqslant n$. Since pos_i $(\sigma)=i$, excsum $(\sigma)=\sum_{i=1}^{n} i=\left(\begin{array}{c}n+1 \\ 2\end{array}\right)$ and $\operatorname{inv}_{\mathrm{B}}(\sigma)=n^{2} \equiv n(\bmod$ $2)$, we have $\operatorname{BDSgnExc}_{n}^{i}(q, t)=t^{i}(-1)^{n} q^{\left(\begin{array}{c}n+1 \\ 2\end{array}\right)}$, completing the proof.

We give a similar bivariate generalization of this result to $\mathfrak{S}_{n}$. Consider the following matrices.

$$
D_{P_{n}}=\left(\begin{array}{ccccc}
0 & q & q & \cdots & q t \\
1 & 0 & q^{2} & \cdots & q^{2} t^{2} \\
\vdots & \vdots & \vdots & \ddots & \vdots \\
1 & 1 & 1 & \cdots & 0
\end{array}\right) \quad D_{P_{n}}^{1}=\left(\begin{array}{ccccc}
0 & q & q & \cdots & q \\
t^{2} & 0 & q^{2} & \cdots & q^{2} \\
\vdots & \vdots & \vdots & \ddots & \vdots \\
t^{n} & 1 & 1 & \cdots & 0
\end{array}\right)
$$

i.e. if $D_{P_{n}}=\left(p_{i, j}\right)_{1 \leqslant i, j \leqslant n}$, then $p_{i, j}$ is obtained by the following procedure: set $p_{i, j}=q^{i}$ if $i<j, p_{i, j}=0$ if $i=j$ and $p_{i, j}=1$ otherwise. After this, if $j=n$, set $p_{i, j}=p_{i, j} \cdot t^{i}$. Likewise, if $D_{P_{n}}^{1}=\left(p_{i, j}^{\prime}\right)$, then, set $p_{i, j}^{\prime}=q^{i}$ if $i<j, p_{i, j}^{\prime}=0$ if $i=j$ and $p_{i, j}^{\prime}=1$ otherwise. After this, if $j=1$, set $p_{i, j}^{\prime}=p_{i, j}^{\prime} \cdot t^{i}$.

Define DSgnExcSum $n(q, t)=\sum_{\pi \in \mathfrak{D}_{n}}(-1)^{\operatorname{inv}_{\mathrm{A}}(\pi)} q^{\operatorname{excsum}(\pi)} t^{\text {pos_n }(\pi)}$ as the bivariate signed excedance-sum enumerator over derangements in $\mathfrak{S}_{n}$. Likewise, define

$$
\operatorname{DSgnExcSum}_{n}^{1}(q, t)=\sum_{\pi \in \mathfrak{D}_{n}}(-1)^{\operatorname{inv}_{\mathrm{A}}(\pi)} t^{\operatorname{pos}_{-} 1(\pi)} q^{\operatorname{exc}(\pi)}
$$


as the bivariate signed excedance enumerator with respect to position of 1 , summed over derangements. We show the following bivariate generalizations of Theorem 19 of [5].

Theorem 28 Let $n \geqslant 2$. Then, DSgnExcSum ${ }_{n}^{n}(q, t)=(-1)^{n-1}\left(\sum_{i=1}^{n-1} t^{i} q^{\left(\begin{array}{c}i+1 \\ 2\end{array}\right)}\right)$ and similarly, DSgnExcSum ${ }_{n}^{1}(q, t)=(-1)^{n-1}\left(\sum_{i=2}^{n} t^{i} q^{\left(\begin{array}{c}i \\ 2\end{array}\right)}\right)$.

Proof: It is clear that $\mathrm{DSgnExcSum} n(q, t)=\operatorname{det}\left(D_{P_{n}}\right)$. After performing the row operation $\operatorname{Row}_{n}:=\operatorname{Row}_{n}-\operatorname{Row}_{n-1}$ and then evaluating the determinant, it is easy to see by induction on $n$ that $\operatorname{det}\left(D_{P_{n}}\right)=(-1)^{n-1}\left(\sum_{i=1}^{n-1} t^{i} q^{\left(\begin{array}{c}i+1 \\ 2\end{array}\right)}\right)$. Similarly, it is clear that $\operatorname{DSgnExcSum}{ }_{n}^{1}(q, t)=\operatorname{det}\left(D_{P_{n}^{1}}\right)$. Performing the column operation $\mathrm{Col}_{n}:=\mathrm{Col}_{n}-\mathrm{Col}_{n-1}$ and then evaluating the determinant, it is easy to see that

$$
\operatorname{det}\left(D_{P_{n}^{1}}\right)=(-1)^{n-1} \sum_{i=2}^{n} t^{i} q^{\left(\begin{array}{l}
i \\
2
\end{array}\right)},
$$

completing the proof.

\section{Acknowledgement}

The Online Encyclopedia of Integer Sequences was consulted for the results in Theorems 15 and 18. We are grateful to Professor N.J.A. Sloane for putting his searchable encyclopedia on the web. Support from project grant P07 IR052, given by IIT Bombay is acknowledged. We thank the referee for a careful reading and for pointing out several places for improvement in this work.

\section{References}

[1] Brenti, F. q-Eulerian polynomials arising from Coxeter groups. Europ. J. Combinatorics 15 (1994), 417-441.

[2] Chen, W., Tang, R., and Zhao, A. F. Derangement Polynomials and Excedances of Type B. Electronic Journal of Combinatorics 16(2) (2009), \#R15.

[3] Mantaci, R. Binomial Coefficients and Anti-excedances of Even Permutations: A Combinatorial Proof. Journal of Combinatorial Theory, Ser A 63 (1993), 330-337.

[4] Mantaci, R., And Rakotondrajao, F. Exceedingly deranging! Advances in Applied Mathematics 30 (2003), 177-188.

[5] Sivasubramanian, S. Signed excedance enumeration via determinants. Advances in Applied Math 47 (2011), 783-794. 